FORMATION Formation emploi

Revue française de sciences sociales

97 | janvier-mars 2007

Former pour dynamiser les territoires

\title{
Les thèses CIFRE, un indicateur de la structuration spatiale de la recherche
}

CIFRE doctoral theses: an index to the spatial structure of research

CIFRE-Dissertationen: Indikatoren für die räumliche Strukturierung der

Forschung

Rachel Levy et Raymond Woessner

\section{(2) OpenEdition}

Journals

Édition électronique

URL : http://journals.openedition.org/formationemploi/1686

DOI : 10.4000/formationemploi.1686

ISSN : 2107-0946

Éditeur

La Documentation française

Édition imprimée

Date de publication : 1 janvier 2007

Pagination : 51-66

ISSN : 0759-6340

Référence électronique

Rachel Levy et Raymond Woessner, « Les thèses CIFRE, un indicateur de la structuration spatiale de la recherche », Formation emploi [En ligne], 97 I janvier-mars 2007, mis en ligne le 31 mars 2009, consulté le 30 octobre 2020. URL : http://journals.openedition.org/formationemploi/1686 ; DOI https://doi.org/10.4000/formationemploi.1686

(c) Tous droits réservés 


\section{Les thèses CIFRE, un indicateur de la structuration spatiale de la recherche}

Par Rachel Levy et Raymond Woessner*

La répartition géographique des thèses en Convention industrielle de formation par la recherche (CIFRE) permet d'établir une cartographie des relations entre les universités et les entreprises. Les dynamiques territoriales de la haute technologie sont dominées par l'île-de-France, même si de nouvelles structures spatiales émergent.

Parmi les différents pays européens, la France constitue un cas particulier pour la science et la technologie (S\&T) car l'État y a joué un rôle historique particulièrement prégnant. Depuis les grands programmes de recherche qu'il a commandités lors des Trente Glorieuses ${ }^{1}$, un territoire national S\&T a été constitué. Il est animé par des communautés de travail pilotées par le haut de la pyramide administrative et politique, avec pour conséquence géographique une hypertrophie de la région parisienne, malgré les politiques de décentralisation actives dès les années 60. Inscrit dans la planification conçue par les grands corps de l'État, le «modèle» français s'est auto-légitimé. Il semblait aller de soi et il n'a guère souffert de contestation jusqu'aux années 80 .

Or, depuis plusieurs décennies, la France évolue dans une contradiction. A priori, le comportement républicain et égalitaire a pu conduire à un saupoudrage des

1 à l'image de l'action du Commissariat à l'énergie atomique, du Centre national des études spatiales ou encore du Plan Calcul.
* Rachel Levy est chargée d'étude au BETA (Bureau d'économie théorique et appliquée), Centre régional associé au Céreq de Strasbourg, et docteur en science économique. Sa thèse, réalisée sous la direction de Jean-Alain Heraud, portait sur l'évaluation de la place de la recherche universitaire dans les systèmes d'innovation /régionaux et nationaux). Ses travaux de recherche ont plus particulièrement trait aux transferts de connaissances et de technologies qui peuvent se réaliser entre une université (et particulièrement l'université Louis Pasteur de Strasbourg) et ses différents partenaires, notamment les partenaires industriels. Elle a notamment publié : "Les doctorants CIFRE : médiateurs entre laboratoires de recherche universitaires et entreprises», Revue d'Économie Industrielle, n 111 , 2005, pp. 79-96; "Le rôle du brevet dans les biotechnologies: Le cas de la BioValley du Rhin Supérieur », en collaboration avec Bureth A., Penin, J. et Wolff S., Éducation et Formation, n 71, 2005. 
Raymond Woessner est maître de conférences, géographe à l'IUFM (Institut universitaire de formation des maîtres) d'Alsace, Strasbourg, membre du CRESAT ICentre de recherches sur les économies, les sociétés, les arts et les techniques), université de Haute-Alsace, Mulhouse, et de la Jeune Equipe 2741 de géographie de l'université Louis Pasteur, Strasbourg. Son domaine d'investigation est l'aménagement du territoire et ses trois dernières publications sont : "Le Grand Est français, une région incomplète», Les Cahiers Scientifiques du Transport, Lyon, 2004, $n^{\circ}$ 46, pp. 45-62. "Les territoires de la Formule 1 », Cahiers Nantais, 2004-2005, n 62-63, pp. 193 à 203. "L'éternel retour du Corridor RhinRhône ", Annales de Géographie n 647, janvier-février 2006, Paris A. Colin, pp. 2-25. Voir hitp:/ / geographiewoessner.apr-strasbourg.org

moyens publics, à l'image des trente et un « incubateurs Allègre », du nom du ministre qui les a créés en 1999. Chaque capitale régionale a ainsi reçu le sien, sauf Lille et Marseille qui en comptent deux, l'Île-de-France cinq (à Pontoise, Cachan et Orsay, et deux à Paris), plus ceux de Grenoble, de Nancy et de Nice. Mais cette politique a été remise en cause par la stratégie européenne de Lisbonne-Göteborg qui cherche à faire émerger des pôles compétitifs au niveau mondial. Il s'agit, dans cette optique, de reconcentrer les moyens sur un nombre limité de pôles, ce qui peut favoriser à nouveau le pôle parisien.

Il est frappant de constater que la littérature française évoquant les problématiques des régions innovantes repose sur un corpus conçu ailleurs, dans les pays anglosaxons en particulier (Veltz, 1995). Le cadre d'analyse des systèmes régionaux d'innovation s'inspire à la fois des notions de districts industriels introduits par Marshall (Zaratiegui, 2004, pour une définition détaillée), du concept de cluster développé par Porter (1998) ou encore de milieu innovateur (Maillat et Kebir, 1999), inscrits dans le cadre théorique de l'économie de la connaissance. "Un système d'innovation peut-être vu comme un ensemble d'acteurs et d'entités tels que des entreprises, mais aussi d'autres organismes et institutions [telles que les universités] qui interagissent pour la génération, l'utilisation et la diffusion de nouvelles connaissances [économiquement rentables »]2 (Fischer,

\footnotetext{
2 Traduction de l'auteur.
}

2000, p. 200). Le caractère innovant d'un système pourra se définir à partir de différents critères parmi lesquels les compétences, la culture de la région (ou de la nation), ses structures institutionnelles et/ou organisationnelles, etc. mais également le nombre d'acteurs actifs dans le système d'innovation ainsi que le nombre de liens reliant les acteurs, et grâce auxquels pourra se développer une réelle interaction. Notons enfin que tout un ensemble d'institutions, les différentes échelles de systèmes d'innovation qui sont analysées dans la littérature (national, régional, sectoriel) ne sont pas indépendantes, mais se chevauchent volontairement. Ainsi la dimension régionale doit notamment se combiner avec la dimension nationale. En d'autres termes, «la dimension régionale est importante mais elle n'est pas exclusive $»^{3}$ (Landabaso et al., 2001, p. 252).

L'objectif de cet article consiste à faire apparaître la structure du territoire français en reprenant la question posée par Joël Mirloup à propos des régions périmétropolitaines et de la métropolisation (Mirloup, 2002) :

- Si le modèle centre/périphérie historique continue sur sa trajectoire séculaire, alors les rapports de «domination » (op. cit., cf. p. 34) l'emportent; dans ce cas, les pôles des différentes régions restent atrophiés par rapport à l'Île-de-France et ne communiquent pas entre eux. La DATAR (Délégation à l'aménagement du territoire et à l'action régionale) a proposé d'intituler ce type de structuration «le scénario néo-jacobin » (DATAR, 2000).

- Ou bien, si un système métropolitain se construit, l'Île-de-France entretient de nombreux rapports avec un certain nombre de pôles relativement puissants et eux-mêmes en relation avec des sous-systèmes régionaux qui deviennent ainsi des systèmes innovants territorialisés.

- Dans tous les cas de figure, le scénario de la DATAR, dit du "local différencié », semble bien improbable : il faudrait dans ce cas que les relations s'établissent de manière primordiale au sein de systèmes régionaux. Le rôle de l'Île-de-France serait alors très estompé et peu structurant.

Pour apporter un éclairage face à cette question, nous utiliserons un indicateur particulier des relations entre laboratoires de la recherche publique et entreprises en

\footnotetext{
${ }^{3}$ Traduction de l'auteur.
} 
France, à savoir les attributions des bourses CIFRE (Convention industrielle de formation par la recherche, (cf. encadré 1). En effet, depuis 1981, la France a développé un outil original permettant aux doctorants de réaliser leur projet de recherche simultanément dans un laboratoire public ${ }^{4}$ et dans une entreprise.

${ }^{4}$ Par souci d'allégement, dans la suite du texte nous utiliserons le terme laboratoire pour désigner les laboratoires académiques et en aucun cas les laboratoires de recherche des entreprises.
Nous présenterons une analyse des échanges de connaissances entre universités et entreprises dans les départements français en nous basant sur l'examen de la localisation des entreprises et des laboratoires qui ont encadré des doctorants CIFRE entre 1982 et 2004. L'analyse de la localisation des entreprises et des laboratoires de recherche impliqués dans des conventions CIFRE permet de construire un indicateur partiel du potentiel de recherche publique

\section{Encadré 1}

\section{Les Conventions industrielles de formation par la recherche (CIFRE) en France}

Les CIFRE permettent de faire collaborer un doctorant, un laboratoire et une entreprise autour d'un même projet. Elles débouchent sur la mise en place d'un projet innovant pour l'entreprise ( ${ }^{*}$ ) et sur une formation industrielle et l'obtention d'un doctorat pour le candidat, mais aussi quelquefois sur l'insertion professionnelle de ce candidat dans l'entreprise où il a effectué la thèse (Quéré, 1994). Ce système a été instauré non seulement pour inciter les entreprises françaises à innover, mais également pour faciliter l'insertion professionnelle des doctorants. Trois types d'acteurs sont impliqués dans une convention CIFRE :

- Via la convention, l'entreprise de droit français va s'engager à mener à bien un projet innovant, en partenariat avec le doctorant et le laboratoire. L'entreprise, outre un engagement financier (salaire minimum de 20215 euros par an pendant trois ans), devra pouvoir fournir au candidat une formation industrielle. En contrepartie, l'entreprise recevra une subvention de I'ANRT (Association nationale de la recherche technique) de 14635 euros par an. Près de la moitié (environ $47 \%$ des cas) des CIFRE sont effectuées en partenariat avec des petites structures (PME indépendantes et filiales de moins de 500 personnes). Les thèses CIFRE passées avec de grandes entreprises sont majoritairement encadrées dans leurs centres de recherche. Les entreprises CIFRE se situent en majorité dans les domaines du matériel électrique et électronique (20\%), mais aussi des services (19\%) et de la parachimie (1 $1 \%$ ). - Le doctorant doit avoir moins de 26 ans, posséder un diplôme français récent de niveau bac +5 ou équivalent, et ne pas encore avoir d'expérience professionnelle. II s'engage à travailler dans l'entreprise en signant un contrat d'embauche et en passant une partie de son temps dans le laboratoire, ce qui lui permettra de rédiger une thèse de doctorat. Les conventions CIFRE ne s'adressent pas uniquement à des doctorants issus de cursus universitaires (ces derniers regroupant plus de $45 \%$ de l'ensemble des doctorants CIFRE). En effet, contrairement aux thèses "classiques », ce système s'adresse aussi à d'autres types de candidats comme les ingénieurs.

- Le laboratoire est implanté dans une université (dans $45 \%$ des cas), une école (37\%) ou un organisme public de recherche en France ( $18 \%$ ) ou à l'étranger (moins de 0,5\% des cas), et il devra assurer une formation de recherche au candidat. Concernant la discipline de la thèse, une grande partie des CIFRE sont effectuées dans les secteurs des sciences et technologies et sciences pour l'ingénieur (45\%), des sciences et structures de la matière (24\%), des sciences de la nature et de la vie (19\%), mais aussi des sciences humaines et sociales (12\%) où les CIFRE se développent notablement, même si elles restent sous-représentées par rapport à l'ensemble des thèses en sciences humaines et sociales soutenues en France $\left({ }^{* *}\right)$. Plus généralement, on peut comparer ces chiffres avec le tableau suivant qui présente la répartition des thèses soutenues en France. On constate notamment une surreprésentation des thèses CIFRE dans le domaine des sciences pour l'ingénieur, puisque le système CIFRE s'adresse plus particulièrement aux doctorants issus d'écoles d'ingénieurs. 
Encadré 1 (suite)

Répartition du total de thèses soutenues par discipline en 2002

\begin{tabular}{|c|c|}
\hline Discipline & \% de thèses soutenues \\
\hline Biologie fondamentale & 5,4 \\
\hline Recherche médicale & 3,9 \\
\hline Biologie appliquée écologie & 5,4 \\
\hline Chimie & 10,0 \\
\hline Physique & 5,7 \\
\hline Sciences de l'univers & 4,0 \\
\hline Sciences pour l'ingénieur & 14,2 \\
\hline Mathématiques & 9,1 \\
\hline Sciences humaines & 25,8 \\
\hline Sciences sociales & 16,6 \\
\hline Total & 100 \\
\hline
\end{tabular}

Source : OST (2004), p. 80.

Ce système est géré au niveau national par l'ANRT. Les données utilisées dans cette étude ont été transmises par cette association qui nous a fourni un fichier sur l'ensemble des 12678 thèses CIFRE, qu'elle a financées entre 1981 et 2004. Ces données concernent à la fois le doctorant (sexe, formation d'origine), le laboratoire d'accueil (domaine de recherche, type institutionnel, code postal) et l'entreprise d'accueil (secteur d'activité, taille de l'entreprise et code postal de l'établissement dans lequel le doctorant CIFRE a séjourné).

(*) : Une étude effectuée par l'ANRT en 2002 a montré que les retombés industrielles des CIFRE se répartissaient de la façon suivante : $14 \%$ de brevets, $17 \%$ de nouveaux produits, $11 \%$ de prototypes mais aussi $19 \%$ de nouveaux procédés et $39 \%$ de savoir-faire.

$\left({ }^{* *}\right)$ : Il nous semble pertinent de considérer les thèses CIFRE en sciences humaines de la même manière que dans d'autres disciplines, car elles permettent de la même façon que les autres thèses CIFRE de faire un lien entre un laboratoire de recherche académique et une entreprise. La différence entre les thèses en sciences humaines et les thèses dans d'autres disciplines, c'est que les thèses en sciences humaines vont pouvoir introduire des innovations organisationnelles et non pas de nouveaux produits (Levy, 2006).

et privée de chacun des départements français. Elle est aussi considérée comme un indicateur des échanges de connaissances entre ces territoires.

Les conventions représentent tout d'abord des échanges de connaissances bilatérales: le transfert de connaissances va du laboratoire à l'entreprise mais aussi de l'entreprise vers le laboratoire. La supervision d'une thèse CIFRE représente à la fois une interaction personnelle entre le doctorant et les représentants de l'entreprise et du laboratoire, et entre les deux institutions. Les échanges de connaissances ainsi générés s'accompagnent d'une production de savoir codifié (la thèse), ainsi que d'un échange de flux financiers (le contrat signé entre le laboratoire et l'entreprise). L'étude des retombées sur les entreprises impliquées montre que ces échanges de connaissances s'accompagnent également d'une création de nouveaux produits ou de l'introduction d'un nouveau savoir-faire dans l'entreprise, dans un certain nombre de cas. En France, d'autres dispositifs de collaboration entre universités et entreprises possèdent également ces caractéristiques. Ainsi, nous pourrions analyser d'autres systèmes d'aide à l'insertion de jeunes diplômés, comme les Conventions de recherche pour les techniciens supérieurs (CORTECHS), les stages de longue durée en PME (petites et moyennes entreprises), ou encore les contrats de recherche entre universités et entreprises. Mais le dispositif de thèse CIFRE est le seul dispositif de ce type qui se déroule sur une période relativement longue (trois ans); cette 
durée permet un échange de connaissances significatif entre les deux institutions et l'élaboration d'un processus de construction collective de savoirs. De plus, en décrivant les types d'entreprises et de laboratoires qui ont participé à l'encadrement de doctorants CIFRE, ainsi que les thèmes de recherche, nous avons observé que le dispositif est globalement assez représentatif ( $c f$. encadré 1 ) de la recherche et de l'industrie françaises. Enfin, d'autres travaux plus qualitatifs (Levy, 2005) nous ont permis de souligner le rôle central du doctorant comme médiateur entre universités et entreprises. En effet, grâce à des allersretours réguliers, il va franchir les distances physiques séparant entreprises et laboratoires. De plus, il va acquérir des connaissances (y compris des connaissances tacites) à la fois dans le laboratoire et dans l'entreprise, qu'il va pouvoir transférer en direction de l'autre partenaire de la collaboration (Levy, 2005). Ces résultats confirment donc la pertinence de l'utilisation d'un tel indicateur pour étudier les réseaux de collaborations entre laboratoires de recherche universitaire et entreprises au sein du système français.

Après la présentation des thèses CIFRE dans l'ensemble du système français, nous chercherons à tester l'existence de sous-systèmes territorialisés dans le nord-est, l'ouest et le sud de la France.

\section{UNE ÉVALUATION CROISSANTE ET INÉGALE DU DISPOSITIF}

Dans un premier temps, nous étudierons la répartition des contrats CIFRE sur le territoire national et son évolution. Jusqu'à quel point l'espace français est-il polarisé ? Les contrats CIFRE s'inscrivent-ils dans un schéma territorial conforme aux inégalités reconnues?

\section{Un système fortement centralisé autour de l'île-de-France}

De 1981 à 2004, plus de 12500 thèses CIFRE ont été commencées. La Figure 1 présente l'évolution du nombre d'inscriptions en thèses CIFRE. On observe que ce système est de plus en plus attractif, puisque en tendance le nombre de CIFRE réalisées s'accroît chaque année, même si on peut observer des paliers dus à l'augmentation des montants financiers accordés par l'État à ces thèses (et plus généralement aux politiques de recherche et d'innovations). En 2004, l'ANRT a d'ailleurs atteint l'objectif affiché de 1000 conventions.

Cette évolution générale recouvre de très fortes disparités au sein du territoire français (Figure 2). Le poids de la Région Île-de-France apparaît déterminant, autant par l'importance de la recherche que par

Figure 1

Évolution des thèses CIFRE (Convention industrielle de formation à la recherche en entreprise) - nombre de CIFRE commencées par an en France

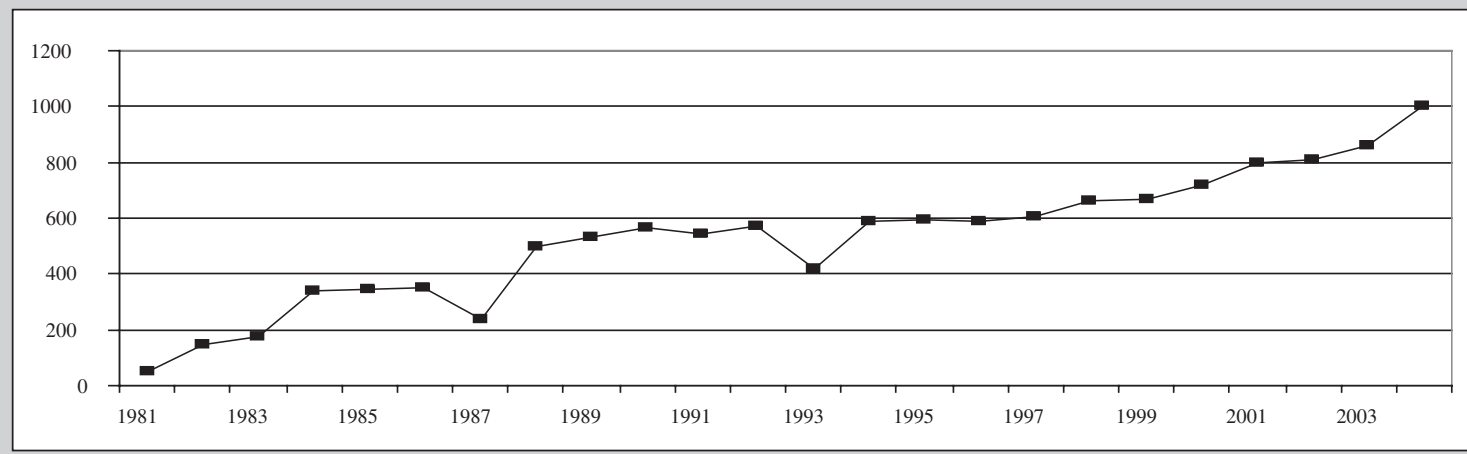

Exemple de lecture : en 2004, plus de 1000 thèses CIFRE ont été commencées.

Source : données ANRT (1981-2004). 12678 CIFRE commencées entre 1981 et 2004. 
celle des relations qu'elle génère. En son sein, les connexions entre Paris et le sud-ouest de son agglomération sont écrasantes. Les contrats CIFRE encadrés en Île-de-France le sont principalement dans des universités parisiennes et dans des entreprises localisées dans le département des Hauts-de-Seine, ainsi que dans les Yvelines et dans l'Essonne.

Avec le reste de la France, la région parisienne entretient des relations «néo-jacobines » de type centrepériphérie : les flux sont principalement connectés sur Paris et les relations interrégionales pèsent très peu. C'est d'abord à Toulouse, Grenoble, Lyon, Marseille et Lille que des pôles secondaires vigoureux et reliés à Paris apparaissent. Lyon se distingue comme étant la seule plate-forme d'interconnexion secondaire du fait de sa proximité relationnelle avec Grenoble et Toulouse. D'autres grandes villes apparaissent nettement plus isolées, à l'image de Marseille, Nice, Bordeaux, ainsi que dans le Grand Ouest et le Grand Est. Notons que certains de ces pôles apparaissent également dans certaines cartes publiées par ailleurs et réalisées sur la base d'autres informations que les CIFRE, comme celles évoquant le nombre d'étudiants et les réseaux de recherches dans les villes européennes au tournant du millénaire (Rozenblat, Cicille, 2003), ou comme les données développées par l'Observatoire des sciences et des techniques concernant par exemple les populations de chercheurs, les publications scientifiques ou les dépôts de brevets (OST, 2004).

\section{Des dynamiques territoriales différenciées}

L'Île-de-France est un cas particulier, ne serait-ce que par l'importance du nombre de contrats CIFRE au sein du total national. Schématiquement, son quart sud-ouest a d'abord démarré, puis sa partie est et nord est montée en puissance, ce qui correspond à l'amorce d'un rééquilibrage voulu de la région parisienne. Sur l'ensemble du territoire hors Paris, on retrouve là aussi des changements relativement conformes aux grandes évolutions. Pour les entreprises, les années 80 et 90 ont d'abord concerné des territoires anciennement industrialisés et revivifiés par la décentralisation des activités de production (au nord de Paris, dans le Grand Est et le Grand Ouest) ; de même, le littoral méditerranéen (Roussillon excepté) est concerné, ce qui confirme la tendance à l'héliotropisme alors évoquée par René Uhrich (1987). Vers 2000 vient davantage le tour de Rhône-Alpes, y compris dans sa bordure du Massif Central, et de la Bretagne et Basse-Normandie, tout comme de la moitié méridionale du Bassin Parisien. Localisés dans les villes d'importance, les laboratoires sont nécessairement moins dispersés que les entreprises. Ils suivent plus ou moins la même tendance, avec le décalage récent vers les pôles universitaires de Rennes, Nantes, Brest, Clermont-Ferrand, Lyon, Grenoble, Chambéry, Montpellier... Une dynamique intéressante se produit également dans le Sud-Ouest, autour de Bordeaux, La Rochelle et Pau.

Les figures 2 et 3 montrent par conséquent des dynamiques territoriales différenciées :

- La métropolisation produit ses effets en Île-deFrance, mais en une vingtaine d'années, la situation a évolué. La périmétropolisation donne sa chance à la portion autrefois délaissée de l'agglomération parisienne, notamment dans sa partie orientale ;

- Des villes moyennes-grandes ou moyennes peuvent tirer leur épingle du jeu, en particulier en Rhône-Alpes, en Bretagne/Loire/Normandie, vers l'Atlantique et les Pyrénées occidentales ;

- Malgré la présence de métropoles régionales, le quart nord-est de la France semble relativement essoufflé. Au nord-ouest de la France, la région Basse-Normandie présente des caractéristiques similaires. «La diagonale aride », c'est-à-dire cet ensemble de régions en déclin démographique et économique qui se regroupent selon une direction nordest/sud-ouest (Brunet, 1994), est une réalité avec quantité de départements mis hors-jeu et avec le vide relatif des régions Auvergne et Limousin, Bourgogne, Champagne-Ardenne.

L'Île-de-France rassemble en permanence 40 à $45 \%$ des entreprises concernées et 25 à $28 \%$ des laboratoires, avec en outre une augmentation de son importance en 2001-2004. Loin derrière l'Île-de-France, la Région Rhône-Alpes monte en puissance et pèse autant que les régions du groupe suivant (ProvenceAlpes-Côte-d'Azur et Midi-Pyrénées qui représentent environ 3 à $5 \%$ du total national). Ce dernier groupe s'affirme également, en particulier dans le 
Figure 2

\section{L'ensemble des échanges entre laboratoires et entreprises, entre 1981 et 2004}

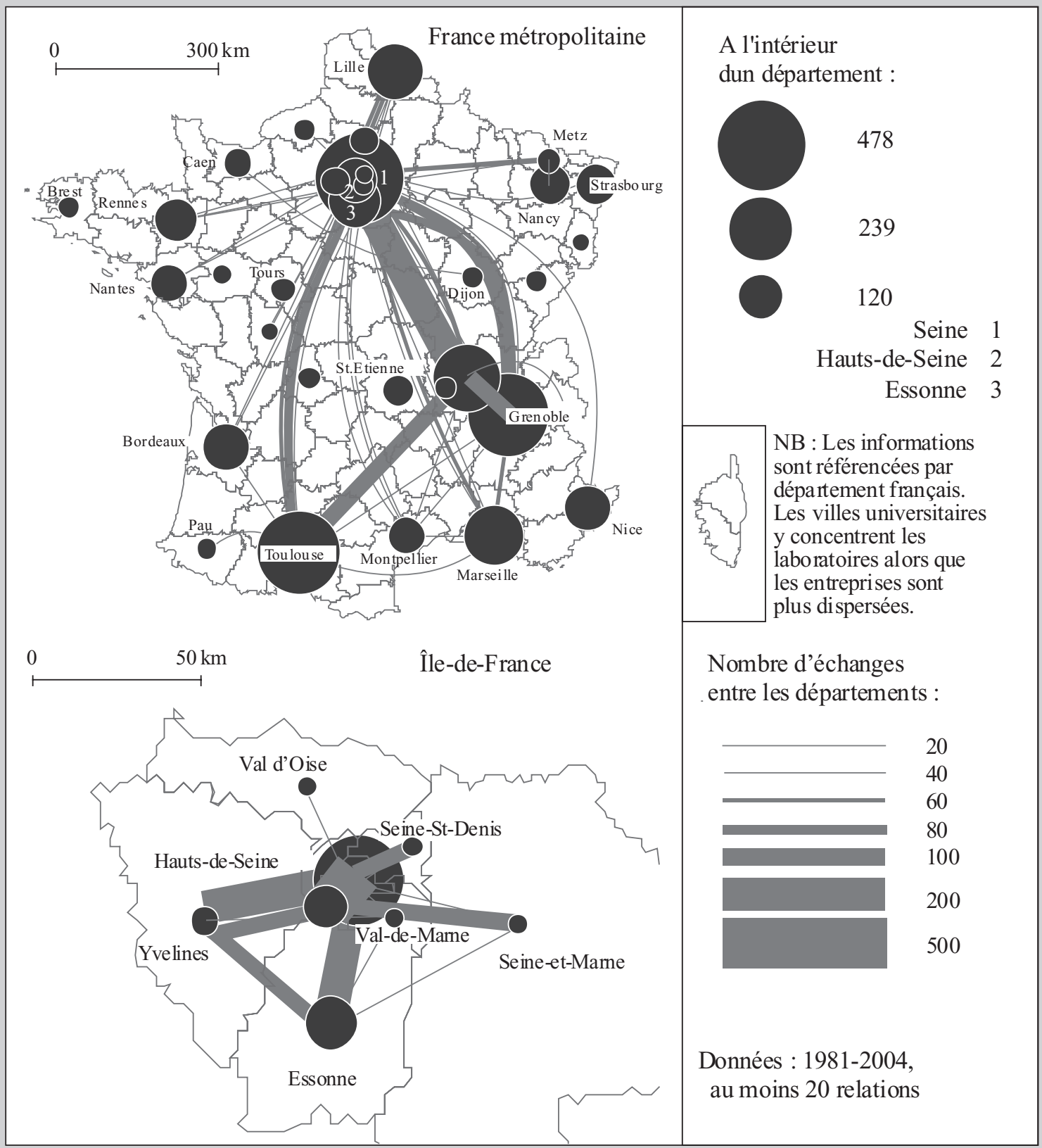

Source : données ANRT (1981-2004).

Logiciel : Corel Draw.

Lecture : ces cartes représentent les liens entre les laboratoires et les entreprises ayant encadré ensemble un doctorant CIFRE (cf. encadré 1). La première carte représente les échanges dans la France entière et la seconde les échanges au sein de la région Île-de-France.

Chaque ligne représente un contrat CIFRE conclu entre un laboratoire et une entreprise localisés dans deux départements différents. Par exemple, entre 1981 et 2004, entre 200 et 500 thèses CIFRE ont été encadrées par un laboratoire universitaire du département de l'Essonne et une entreprise parisienne ou par un laboratoire universitaire parisien et une entreprise de l'Essonne. Le score maximal est atteint entre la Seine et les Hauts-de-Seine avec environ 500 relations CIFRE.

Chaque point représente un contrat CIFRE passé entre un laboratoire et une entreprise localisés dans le même département. Par exemple, plus de 239 CIFRE ont été encadrés par un laboratoire de recherche de l'Essonne et une entreprise du même département. 


\section{Figure 3}

\section{Profils et évolutions du nombre de CIFRE encadrées par les laboratoires et les entreprises des régions françaises}
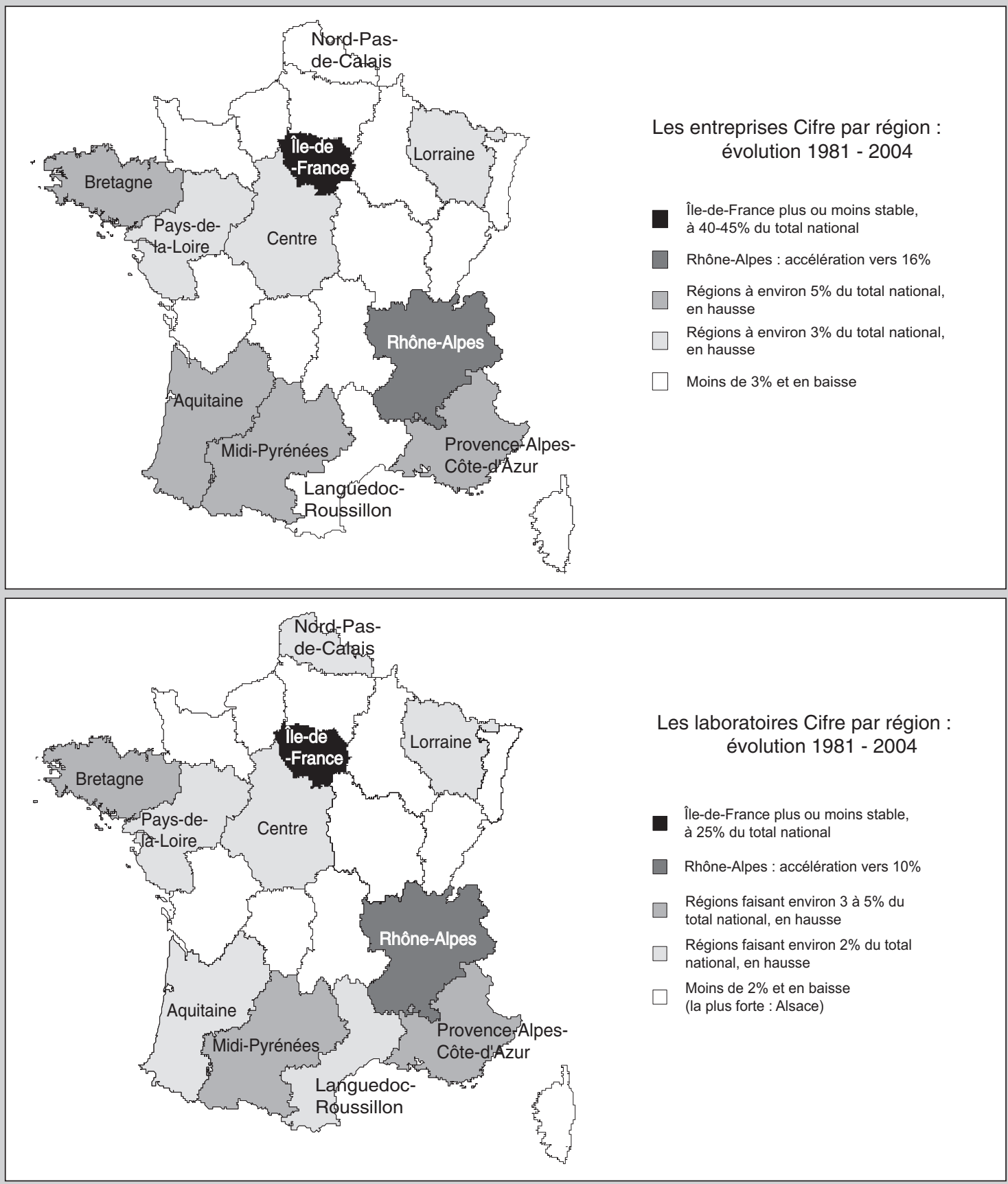

Source : données ANRT (1981-2004, divisées en cinq périodes inégales)

Logiciels : Excel, Corel Draw.

Lecture : les cartes représentent le pourcentage maximum de thèses encadrées dans un territoire et l'évolution qui l'accompagne, entre 1981 et 2004. La première carte représente le nombre de CIFRE encadrées dans les entreprises du département ; la seconde, les CIFRE encadrées par les laboratoires du département. 
sud et l'ouest, grâce aux laboratoires des grandes villes universitaires. Les régions en difficultés, à la fois très peu et de moins en moins impliquées, dessinent une sorte de fer à cheval péri-francilien auquel l'ouest échappe. Tout à l'est, la Lorraine résiste, alors que l'Alsace sort peu à peu du système, en particulier au niveau de ses laboratoires. En effet, en termes de recherche universitaire, la région Alsace est centrée autour de l'université Louis Pasteur de Strasbourg et occupe une place particulière dans la région. Elle s'insère dans des réseaux de recherche publique mais aussi privée de dimension internationale (plus que nationale) et particulièrement dans le secteur de la chimie et de la biologie (Levy, 2005). Au total, la métropolisation, l'héliotropisme et la décentralisation semblent conjuguer leurs effets et provoquer des discriminations territoriales.

\section{DES SOUS-SYSTÈMES RÉGIONAUX QUASI INEXISTANTS}

Sur la base de ces évolutions, et malgré la permanence de la prééminence de l'Île-de-France, existe-t-il néanmoins des systèmes régionaux identifiables à leur échelle? L'enjeu est significatif pour plusieurs raisons : des pôles de compétitivité S\&T peuvent-ils ou sont-ils en train d'émerger? Sont-ils polarisés par une métropole régionale ou bien se structurent-ils de manière polynucléaire sur la base d'un réseau régional ou interrégional ? Apparaissent-ils relativement autonomes par rapport à l'Île-de-France (ce qui semble $a$ priori constituer une gageure) ou bien peuvent-ils se développer adossés aux ressources franciliennes?

Dans ses travaux prospectifs, la DATAR avait identifié plusieurs scénarios de croissance et de structuration régionales (DATAR, 2000). Celui du «polycentrisme maillé », qui ne laisse pas de portion du territoire national en déshérence, peut être écarté : on en a déjà constaté le caractère improbable. Celui de «l'archipel éclaté », où les grandes villes françaises, proches des frontières nationales, développent les villes de leur zone d'influence régionale tout en nouant des coopérations avec leurs homologues étrangères proches, ne peut pas être pris en compte ici, puisque les CIFRE fonctionnent quasi-exclusivement dans le cadre national (à l'exception de 79 thèses encadrées entre un laboratoire étranger et une entreprise française). Il reste deux scénarios appropriés à notre recherche :

- Le «centralisme rénové» ou le «néojacobinisme ». L'île-de-France reste la maîtresse du jeu ; dans son sillage, des pôles se développent au gré des aléas et des opportunités ;

- Le « local différencié ». Des grappes de villes se structurent à plusieurs échelles : entre elles, au niveau national et international. Elles laissent subsister des vides interstitiels importants.

Le tableau 1 présente trois macro-régions, le Nord et l'Est, l'Ouest, le Sud, qui forment ici trois ensembles légitimement séparés parce qu'ils ne communiquent quasiment pas entre eux en termes de contrats CIFRE. En revanche, tous trois se connectent fortement sur la région Île-de-France qui, en soi, forme un quatrième territoire.

\section{Tableau 1}

La destination des CIFRE (contrats issus des entreprises du nord-est, de l'ouest et du sud de la France)

\begin{tabular}{|c|c|c|c|}
\hline & En interne & Vers 1'Île-de-France & Total \\
\hline Nord et Est & 725 & 19 & 744 \\
\hline Ouest & 104 & 315 & 419 \\
\hline Sud & 786 & 1034 & 1820 \\
\hline
\end{tabular}

Source : données ANRT (1981-2004). 2983 CIFRE issues des entreprises du nord-est, de l'ouest et du sud de la France Seules les données > 10 par département sont prises en compte sur la période 1981-2004.

Lecture : 104 CIFRE ont été encadrées par une entreprise localisée dans l'ouest de la France et un laboratoire implanté dans l'ouest, et 315 CIFRE ont été encadrées par une entreprise localisée dans l'ouest de la France et un laboratoire de recherche d'Ile-de-France. 


\section{Une faible intégration du nord et de l'est}

Pour le grand quart nord-est de la France, force est de constater qu'il n'existe pas de système interrégional (ou interdépartemental) pour deux raisons principales :

- Les relations externes l'emportent largement: les entreprises de la région parisienne alimentent les flux de manière écrasante vers le nord et l'est de la France.

- Les laboratoires de Nancy, Lille et Strasbourg travaillent majoritairement avec les entreprises de leur département. Besançon et Dijon restent de petits pôles.
La Figure 4 montre l'importance relative des pôles et des relations du quart nord-est, en interne comme en externe, en partant de la localisation des entreprises vers les laboratoires situés dans différents départements.

Autrement dit, pour faire prospérer les relations entre les laboratoires et les entreprises, mieux vaut exploiter des synergies avec Paris plutôt qu'avec ses « égaux » de l'est ou du nord. Nancy et la Meurthe-et-Moselle illustrent cette stratégie de manière particulièrement intense. Au contraire, Strasbourg préfère le national,

\section{Figure 4}

\section{Des sous-systèmes improbables au nord et à l'est}

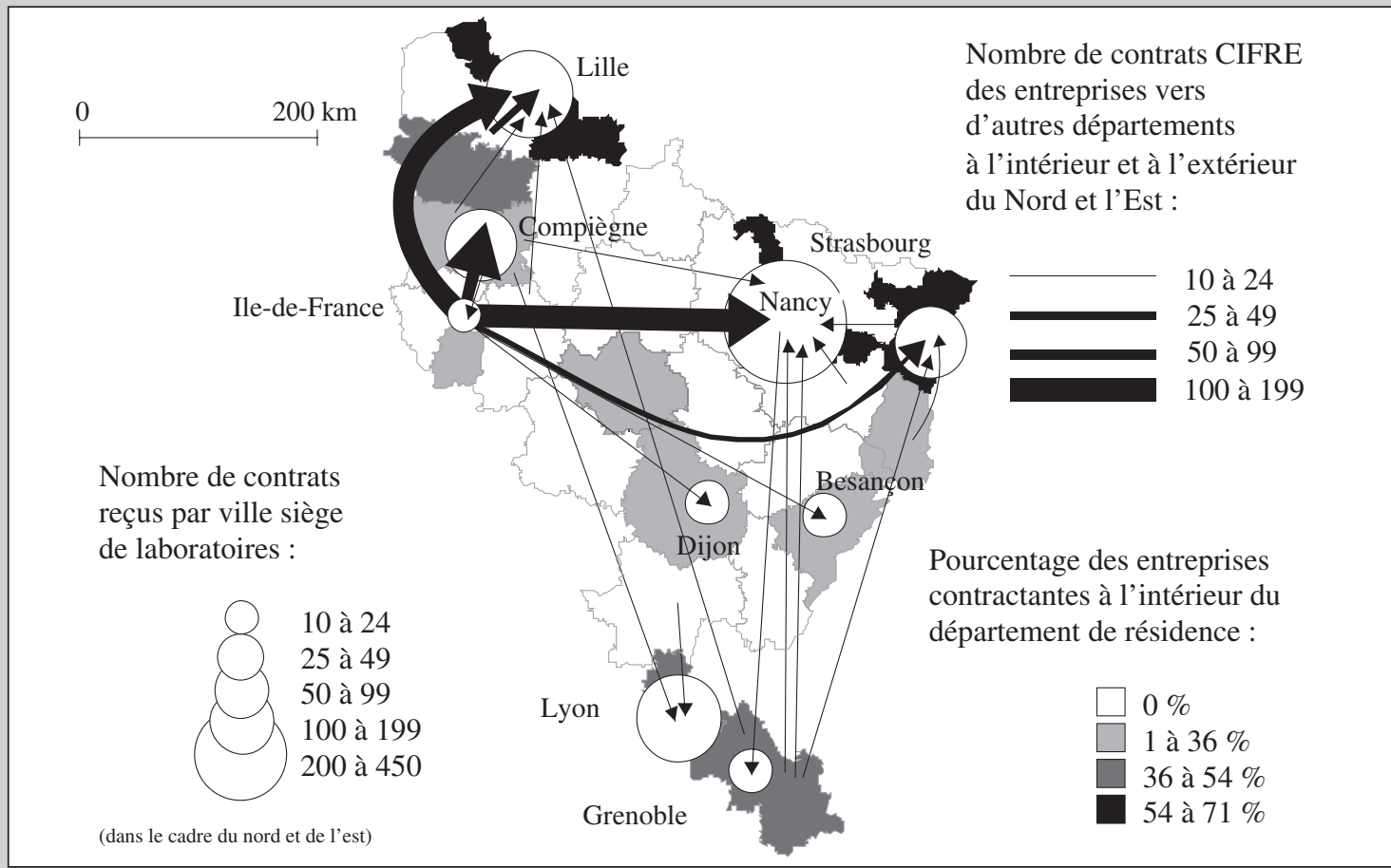

Source : données ANRT (1981-2004). 1737 CIFRE encadrées dans une entreprise du nord-est de la France (Alsace, Bourgogne, Champagne-Ardenne, Franche-Comté, Nord, Lorraine, Picardie).

Logiciel : Corel Draw.

Lecture : cette carte représente les liens entre les laboratoires et les entreprises ayant encadré ensemble un doctorant CIFRE ( $c f$. encadré 1). Les flèches représentent le nombre de thèses encadrées par une entreprise d'un département et un laboratoire de recherche d'un autre département. Les cercles représentent le nombre de thèses encadrées dans un laboratoire de recherche du département. Le niveau de gris des départements représente le pourcentage de thèses encadrées par une entreprise et un laboratoire de recherche localisés dans le même département, même si sur la carte n'est pas indiqué le nom du département mais le nom de la principale ville du département (ville accueillant la majorité des CIFRE du département).

Exemple de lecture : entre 100 et 199 CIFRE ont été encadrées dans un laboratoire de recherches localisé dans l'agglomération de Strasbourg. Entre 1 et $36 \%$ des thèses CIFRE encadrées dans une entreprise côte-dorienne (autour de Dijon) ont été co-encadrées dans un laboratoire de recherche de l'agglomération de Dijon. 
voire l'international à l'interrégional. Il subsiste néanmoins un fait important pour la constitution d'un éventuel système régional: si les entreprises régionales ne se tournent guère vers la région parisienne, c'est probablement parce qu'elles préfèrent les relations de proximité.

Les Pôles de compétitivité, qui sont souvent interrégionaux, pourront-ils stimuler cette dynamique de proximité ? A priori, les éléments de réponse apparaissent peu encourageants. Dans le cas de la macro région du Nord et de l'Est, on ne dénombre que 4 pôles interrégionaux sur un total de 13 au niveau national. Ainsi, le Pôle «Véhicule du Futur», qui associe l'Alsace et la Franche-Comté, devra précisément dépasser la fracture culturelle qui sépare les deux régions (Lévy, Woessner, 2001). En 2006, les premières actions entreprises par ce Pôle se situent de préférence soit en Alsace, soit en Franche-Comté, soit en réunissant des partenariats de type AlsaceAllemagne ou Franche-Comté-Suisse. Au contraire, du fait même de leur structuration nationale (ce système est géré de façon centrale par l'ANRT), les bourses CIFRE pourraient constituer un levier pour l'intégration interrégionale des Pôles en général.

\section{Vers une Sun Belt 5 française?}

L'examen de la répartition des contrats CIFRE montre le déplacement vers le sud des activités de haute technologie (Figure 5). Cette dynamique semble vouloir s'accélérer, en tout cas se consolider (Figure 3). Toulouse, Montpellier, Lyon et Grenoble constituent des pôles majeurs, forts de leur relation entreprises/laboratoires in situ et de leur ouverture vers l'Île-de-France, ainsi que vers les autres pôles situés « au sud de la Loire ». Toutefois, il n'existe pas de système méridional intégré, de scénario de type «local différencié » (de connexions fortes entre les villes d'un même bassin régional) pour reprendre l'expression de la DATAR, du fait de l'asymétrie ou de l'absence de certaines relations :

- Ce sont toujours les relations entre les entreprises franciliennes et les différents pôles universitaires qui

\footnotetext{
${ }^{5}$ La Sun Belt (en français la « ceinture du soleil ») se compose des États du sud et de l'ouest des États-Unis présentant un dynamisme économique et un cadre de vie agréable.
}

l'emportent dans tous les cas de figure, avec une mention particulière pour Grenoble ;

- Les relations interrégionales se font principalement dans des territoires restreints. Ainsi, RhôneAlpes s'affirme en tant que territoire réticulaire autour de Grenoble, de Lyon et, pour une moindre part, de Saint-Etienne. À travers leur politique prospective, qui vise à développer et à partager l'économie de la connaissance, ces villes cherchent généralement à favoriser ce type d'articulation. Un deuxième groupe concerne Toulouse, Montpellier, Marseille et Grenoble principalement, où un territoire interrégional semble vouloir se dessiner ;

- Bordeaux, Nice et Clermont-Ferrand apparaissent mal connectées à cette structure, au profit de relations privilégiant d'abord l'Île-de-France ;

- La Corse est absente de la carte. Cela s'explique notamment en raison de sa faible intensité d'investissement de recherche et développement ${ }^{6}$.

\section{L'ovest français face à la primauté francilienne}

Depuis plusieurs décennies, la problématique de 1'Arc atlantique a été énoncée par les géographes (Brunet, 1997). Les villes et les régions atlantiques sont traditionnellement connectées à Paris mais elles souffrent de la faiblesse de leurs relations en interne. Les réseaux de transport illustrent cette structuration, avec le tracé des autoroutes et des lignes des TGV (train à grande vitesse), qui sont autant de «flèches » reliant ces régions à la capitale nationale. Les régions atlantiques avaient tenté une intégration avec, par exemple, le programme européen "Atlantis », qui aurait dû développer le cabotage maritime, mais l'opération a tourné court; elles attendent à présent une forme d'intégration grâce à l'autoroute dite des « estuaires", qui relie la Seine, la Loire et la Garonne. La Figure 6 s'inscrit parfaitement dans cette problématique. On y voit les flèches parisiennes, «tirées » vers les métropoles régionales (à l'exception de Caen, à peu près absente du jeu et donc de la carte). En outre, Rennes, Poitiers et

${ }^{6}$ Ainsi l'Observatoire des sciences et des techniques précise que "le total [de différents indicateurs de R\&D] pour la France inclut les données de la Corse et des Dom-Tom, mais ces deux régions ne figurent pas dans le tableau en raison de leur faible activité de $R \& D »(\mathrm{OST}, 2004)$. 


\section{Des Midis puissants à l'intégration incomplète}

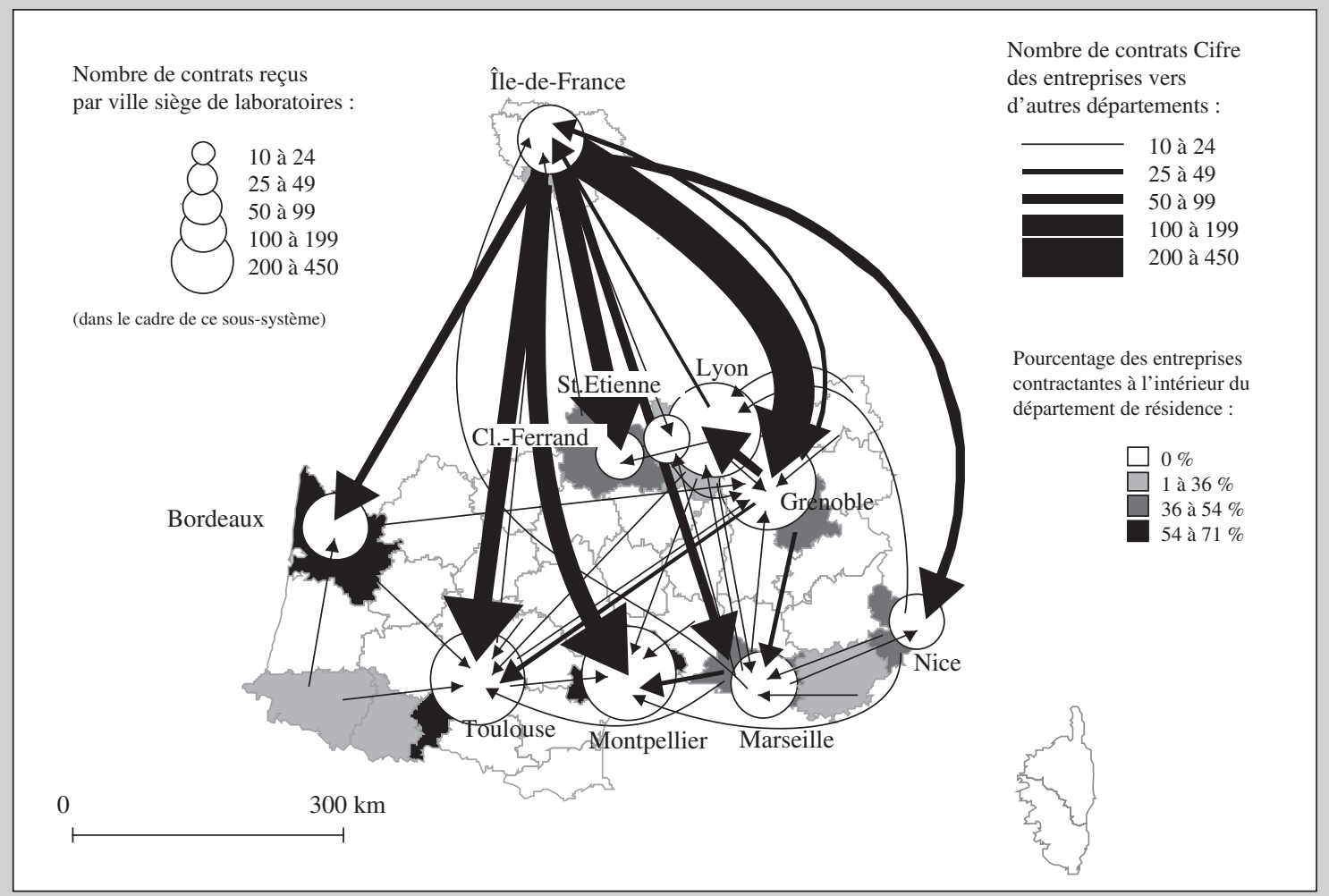

Source : données ANRT (1981-2004). 3953 CIFRE encadrées dans une entreprises du sud de la France (Aquitaine, Auvergne, Languedoc-Roussillon, Limousin, Midi-Pyrénées, Provence-Alpes-Côte d’Azur, Rhône-Alpes).

Logiciel : Corel Draw.

Lecture : cette carte représente les liens entre les laboratoires et les entreprises ayant encadré ensemble un doctorant CIFRE ( $c f$. encadré 1). Les flèches représentent le nombre de thèses encadrées par une entreprise d'un département et un laboratoire de recherche d'un autre département. Les cercles représentent le nombre de thèses encadrées dans un laboratoire de recherche du département. Le niveau de gris des départements représente le pourcentage de thèses encadrées entre une entreprise et un laboratoire de recherche localisés dans le même département, même si sur la carte n'est pas indiqué le nom du département mais le nom de la principale ville du département (ville accueillant la majorité des CIFRE du département).

Exemple de lecture : entre 10 et 24 CIFRE ont été encadrées par une entreprise localisée autour de Bordeaux et un laboratoire de recherches implanté autour de Toulouse. Entre 200 et 450 CIFRE ont été encadrées dans un laboratoire de recherches localisé en Gironde (autour de Bordeaux). Entre 54 et $71 \%$ des thèses CIFRE encadrées dans une entreprise girondine ont été co-encadrées dans un laboratoire de recherche localisé autour de Bordeaux.

Limoges fonctionnent d'abord sur leur propre territoire, alors que Nantes et la Haute-Normandie s'appuient sur des ressources externes.

La configuration de la Bretagne est particulièrement attendue car elle se distingue par son articulation réticulaire connue pour être robuste, du fait des développements endogènes, effectifs dès les années 50 (Phlipponneau, 2002). Par la suite, le technopôle de Rennes a développé une articulation réticulaire avec d'autres villes bretonnes et de l'ouest en général (Benko, 1993). La Figure 6 montre qu'effectivement la Bretagne est la seule région de l'ouest à connaître une certaine intégration, notamment entre les pôles de Rennes et de Brest, même si les relations avec Paris restent prédominantes. En revanche, en Bretagne comme ailleurs, les contrats CIFRE n'assurent pas de liaison avec les autres régions de l'Arc atlantique. 


\section{Figure 6}

\section{La faiblesse des relations régionales de l'ouest}

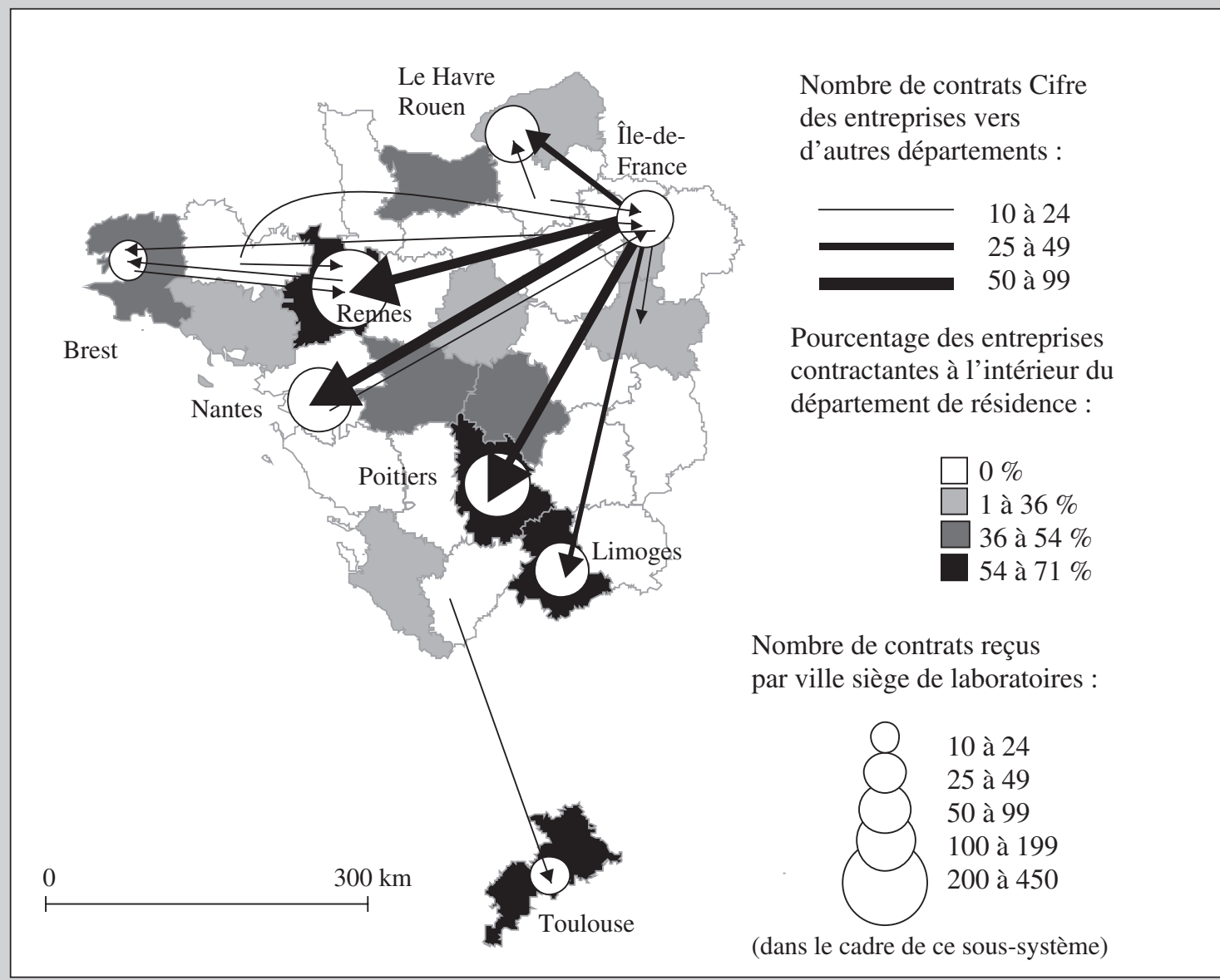

Source : Données ANRT (1981-2004). 1120 CIFRE encadrées dans une entreprises de l'ouest de la France (Basse Normandie, Bretagne, Centre, Haute-Normandie, Pays de Loire, Poitou-Charentes).

Logiciel : Corel Draw.

Lecture : Cette carte représente les liens entre les laboratoires et les entreprises ayant encadré ensemble un doctorant CIFRE (cf. encadré 1).

Les flèches représentent le nombre de thèses encadrées par une entreprise d'un département et un laboratoire de recherche d'un autre département. Les cercles représentent le nombre de thèses encadrées dans un laboratoire de recherche du département. Le niveau de gris des départements représente le pourcentage de thèses encadrées par une entreprise et un laboratoire de recherche localisés dans le même département, même si sur la carte n'est pas indiqué le nom du département mais le nom de la principale ville du département (ville accueillant la majorité des CIFRE du département).

Exemples de lecture : entre 10 et 24 CIFRE ont été encadrées par une entreprise localisée dans le Finistère et un laboratoire de recherches implanté autour de Rennes. Entre 25 et 49 CIFRE ont été encadrées dans un laboratoire de recherches localisé dans le Finistère (autour de Brest). Entre $36 \%$ et $54 \%$ des thèses CIFRE encadrées dans une entreprise du Finistère (autour de Brest) ont été co-encadrées dans un laboratoire de recherche implanté autour de Brest. 
L'utilisation des CIFRE pour établir une cartographie dynamique des relations entre les universités et les entreprises au sein de l'espace français (pris dans sa totalité, dans des macro régions ou encore à une échelle plus fine) corrobore les résultats évoqués par d'autres publications. Les CIFRE apparaissent certes comme des signaux limités, mais qui révèlent néanmoins un certain nombre d'éléments clés. À la lumière de cette recherche, trois aspects utiles à la compréhension des dynamiques territoriales de la haute technologie méritent d'être soulignés: la distance entre les acteurs, le rôle et la place de l'îlede-France, ainsi que les éléments pouvant introduire une nouvelle forme de territorialisation.

La cartographie des CIFRE confirme la géographie des centres de recherche français et un certain nombre de faits avérés par ailleurs, comme la prééminence du système national d'innovation piloté par le système d'innovation de l'Île-de-France et, partant, la faiblesse des intégrations territoriales, malgré une relative émergence de pôles secondaires, dans le Midi en particulier. La primauté parisienne reste donc incontestée, à l'image du scénario du «centralisme rénové» de la DATAR (l'Île-de-France reste la maîtresse du jeu, même si elle substitue des relations de type contractuel à son autorité traditionnelle).

Toutefois, Grenoble semble vouloir constituer un second pivot au sud de la France, avec de fortes relations vers Lyon, Toulouse, Montpellier et Marseille, elles-mêmes relativement interconnectées. Ailleurs, les relations interrégionales sont à la peine, ou franchement inexistantes. C'est pourquoi les régions de l'est et du nord ne forment pas de systèmes intégrés et continuent à collaborer avec l'île-de-France. À l'exception de la Bretagne, les entreprises de l'ouest se tournent également vers l'Île-de-France. Ainsi, la compétitivité des centres secondaires du territoire national est interpellée : peuvent-ils prospérer sur la base des seules relations internes entre laboratoires et entreprises? Ou bien, faute de système régional, doivent-ils se connecter sur l'Île-de-France? En termes de prospective régionale, les enjeux apparaissent stratégiques pour les différentes régions comme pour l'ensemble du système territorial français. La plupart des territoires ont été évincés dans la compétition qui régit l'innovation et la recherche. Un nombre limité de pôles s'est installé dans le paysage.

Les CIFRE jouent un rôle structurant dans les distances qui séparent les acteurs. La notion même de distance se situe au cœur de la problématique des réseaux. Malgré le raccourcissement de la distancetemps ou de la distance-coût pour tous les types de flux, la proximité physique reste une donnée fondamentale: comme nous le voyons dans les figures $\mathbf{1}$, et $\mathbf{4}$ à $\mathbf{6}$, de nombreux contrats CIFRE sont effectués au sein du même département et l'emportent sur les relations avec l'extérieur. D'un autre côté, la distance physique qui sépare les contractants est plus facilement vaincue lorsque l'Île-de-France est concernée. Cette double réalité - proximité locale et proximité parisienne - pèse lourdement sur l'émergence de systèmes régionaux, qui apparaissent entravés par plus petit et plus grand qu'eux.

La relation entreprise/université, à travers les thèses CIFRE, révèle (comme pourraient le faire d'autres indicateurs) la territorialisation. Celle-ci consiste en l'émergence de nouvelles structures spatiales autour d'éléments appartenant à divers pôles et mis en relation. En établissant des relations entre les chercheurs et les institutions, les contrats CIFRE illustrent des phénomènes structurels. S'agit-il de l'émergence de nouveaux systèmes territoriaux ou de la transformation des relations entre entreprises et universités? Le système CIFRE permet d'instaurer des relations de collaboration de moyen terme (trois ans), à distance (le doctorant permettant de faire le pont entre universités et entreprises même localisées dans deux régions éloignées) et dans des secteurs d'activités et des domaines de recherche plus variés que les domaines d'excellence des pôles territoriaux. De telles formes de coopération sont donc à encourager car elles peuvent créer de petits vortex ${ }^{7}$ pratiquement à différentes échelles, et en particulier aux niveaux inter et intrarégionaux.

\footnotetext{
${ }^{7}$ Un vortex est une interprétation picturale spiroïde humaine tendant à montrer une description de l'infini, généralement composante élémentaire de la notion de fractal. L'objectif de cette image est de montrer que, tout comme dans un fractal, les relations entre acteurs de la recherche qui existent au niveau national peuvent être reproduites au niveau intrarégional ou interrégional.
} 
Bibliographie

ANRT (2002), 1981/2001, 20 ans de CIFRE, Dossier réalisé par l'ANRT à l'occasion des 20 ans du système CIFRE.

Asheim B.T., Isaksen A. (2002), « Regional innovation systems: the integration of local "Sticky" and global "ubiquitous" knowledge », Journal of Technology Transfer, 27, pp.77-86.

Benko G. (1993), Géographie des technopôles, Paris, Masson, $223 \mathrm{p}$.

Brunet R. (1994), La France, un territoire à ménager, Paris, Éditions n ${ }^{\circ}$, 327 p., cf. p. 114.

Brunet R. (1997), Territoires de France et d'Europe, Paris, Belin, 320 p.

Bureth A., Heraud J.A. (2001), « Institutions of Technological Infrastructure (ITI) and the generation and diffusion of knowledge », in K. Koschatzky, M. Kulicke, A. Zenker (Eds.), Innovation networks, Physica Verlag, Heidelberg: Springer, pp. 69-91.

DATAR (2000), Aménager la France de 2020, Paris, La Documentation française, $87 \mathrm{p}$.

Fischer M.M. (2000), « Innovation, knowledge creation and systems of innovations ", Annals of Regional Science, 35, pp.199-216.

Koschatzky K. (2004), « The role of R\&D services in managing regional knowledge generation - a regional differenciation », in Karlson C., Flensburg, P., Horte S.A. (Eds), Knowledge spillovers and knowledge management, Edward Elgar, pp. 237-267.

Landabaso M, Oughton C., Morgan K. (2001), «Innovation Networks and Regional Policy in Europe », in Koschatzky K., Kulicke M., Zenker A. (Eds.), Innovation Networks- Concepts and Challenges in the European Perspective, Heidelberg: Physica, pp. 243-273.
Levy C., Woessner R. (2001), «Les entreprises en zone de marge : l'exemple de Rhin-Sud » in Groupe de recherche RITMA, Regards croisés sur les territoires de marge(s), sous la direction de J.-A. Héraud et H. Nonn, Maison des Sciences de l'Homme de Strasbourg, Presses Universitaires de Strasbourg, pp. 147 à 176.

Levy R. (2005), «Les doctorants CIFRE : médiateurs entre laboratoires de recherche universitaires et entreprises ", Revue d'Économie Industrielle, volume 111, pp. 79-96.

Levy R. (2006), "The CIFRE PhDs: A Tool for Mediation between Laboratories and Firms in the Humanities and Social Sciences », in G. Krücken, A. Kosmützky, M. Torka (Eds.), Towards a Multiversity? Universities between Global Trends and National Traditions, pp. 217-234.

Maillat D., Kebir L. (1999), «Learning région et systèmes territoriaux de production », Revue d'Economie Régionale et Urbaine, 3, pp. 429-448.

Mirloup J. (Dir.) (2002), Régions périmétropolitaines et métropolisation, Collection du CEDETE, Presses Universitaires d'Orléans, 303 p.

Muller E. (2001), Innovation interactions between Knowledge-Intensive Business Services and Small and Medium-Sized Enterprises, Heidelberg: Physica Verlag, Springer.

OST (2004), Rapport de l'observatoire des sciences et technologies, (Barré, Esterlé, Éds.) Paris, Économica.

Oughton C., Landabaso M., Morgan K. (2002), «The regional innovation paradox: innovation policy and industrial policy», Journal of Technology Transfer, 27, pp.97-110.

Phlipponneau M. (2002), « Industrie et aménagement du territoire, essai de périodisation », in Caro P., Dard O., Daumas J.-C. (Dir.), La politique 
d'aménagement $d u$ territoire, racines, logiques et résultats, Presses Universitaires de Rennes, Collection « Espace et Territoires », pp. 103 à 120.

Porter M. (1998), «Clusters and the new economics of competition », Harvard Business Review, NovDec, pp. 77-90.

Quéré M. (1994), «The "convention CIFRE": a successful French incentive scheme for the management of human resources in research activity », International Journal on Technology Management, 9 , pp. 430-439.

Rozenblat C., Cicille P. (2003), Les villes européennes, Paris, La Documentation française DATAR, $94 \mathrm{p}$.
Strambach S. (2001), « Innovation Processes and the Role of Knowledge-Intensive Business Services (KIBS) », in Koschatzky K., Kulicke M., Zenker A. (Eds): Innovation networks - concepts and challenges in the European perspective, Heidelberg/New York: Physica-Verlag, pp. 53-68.

Uhrich R. (1987), La France inverse? Paris, Économica, $390 \mathrm{p}$.

Veltz P. (1995), Mondialisation, villes et territoires, Paris PUF, 2e édition 2005, 288 p.

Zaratiegui J.-M., (2004), «Marshallian industrial districts revisited», Problems and Perspectives in Management, février-mars-avril, pp. 80-97, pp. 4358 et $49-62$.

\title{
Résumé
}

\section{Les thèses CIFRE,}

\section{un indicateur de la structuration spatiale de la recherche}

\author{
Rachel Levy et Raymond Woessner
}

En matière d'innovation, la notion de réseau public-privé joue un rôle essentiel. En ce sens, les thèses CIFRE (Convention industrielle de formation par la recherche) en entreprise permettent de mesurer une forme de relation entre les entreprises et les laboratoires universitaires. II en résulte un maillage du territoire français qui apparaît particulièrement déséquilibré. L'agglomération parisienne constitue le centre principal, alors que les autres villes n'occupent que des positions secondaires. Toutefois, des grappes urbaines semblent émerger, sur la base de relations bi-ou multilatérales, en complément de leur articulation avec Paris.

Mots clés

Recherche-développement, thèse, entreprise, répartition géographique Journal of Economic Literature : I 23, J 61, O 32 